\title{
Parametric Comparative Analysis of Underwater Wireless Sensor Networks Routing Protocols
}

\author{
Parul Garg \\ Department of Computer Science and Engineering \\ Guru Nanak Dev University \\ Amritsar, India
}

\author{
Sandeep Waraich \\ Department of Computer Science and Engineering \\ Guru Nanak Dev University \\ Amritsar, India
}

\begin{abstract}
The underwater wireless sensor networks have found many applications in today's world as they are used in medical applications, sea exploration, military applications and many more. With the advancement in the underwater wireless sensor networks technology, new research challenges are found that are to be resolved like how efficient routing can be done without sacrificing energy consumption of the sensor nodes, how the deployment of the sensor nodes should be done and so on. Due to the movement of sensor nodes with the water currents, the deployment and routing becomes a difficult task. In this paper, various routing protocols like Information Carrying routing protocol, Depth Based routing protocol, Constraint Based Depth based routing protocol, Directional flooding routing protocol are discussed and a comparative analysis of these routing protocols on the basis of various parameters like localization information, network topology, and use of control packets, network architecture used is presented.
\end{abstract}

\section{General Terms}

Underwater Wireless Sensor Networks, Routing Procedures, Network Architecture

\section{Keywords}

Depth based routing, location awareness, acoustic nodes, dynamic addressing.

\section{INTRODUCTION}

The most recent improvements in remote interchanges, and computerized hardware lead to the development of sensor nodes that are little in size and interconnect in short separations. These modest sensor nodes that comprises of sensing, information preparing, and conveying parts, impact the thought of sensor networks [1]. A wireless sensor system (WSN) is made of a huge number of sensor nodes that are thickly sent in an unattended domain. The improvements taken in sensing innovation, low-control microcontrollers and correspondence radio have energized the large scale manufacturing of generally economical sensor nodes [24]. The beneath figure demonstrates the essential structural planning of the $2 \mathrm{D}$ wireless sensor systems.

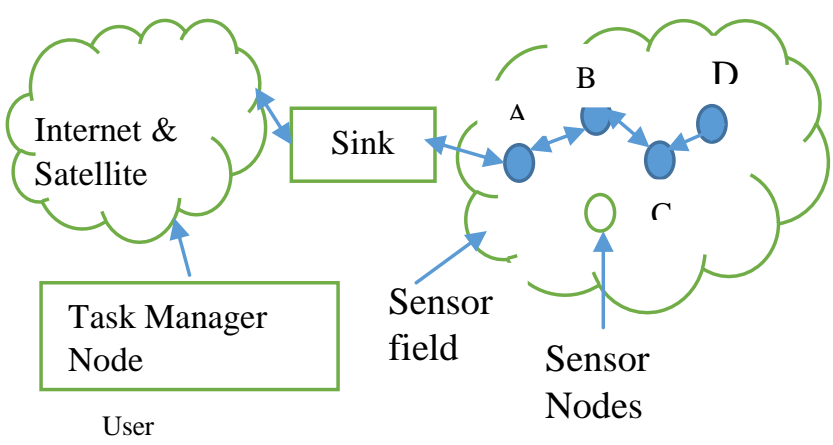

Figure 1 Sensor nodes deployed in sensor environment

\subsection{Components of a Node}

The basic components of a node are shown in figure 2: a sensing unit comprising of sensor unit and ADC (Analog to Digital Converter), CPU (Central processing unit), power unit and transceiver unit [3].

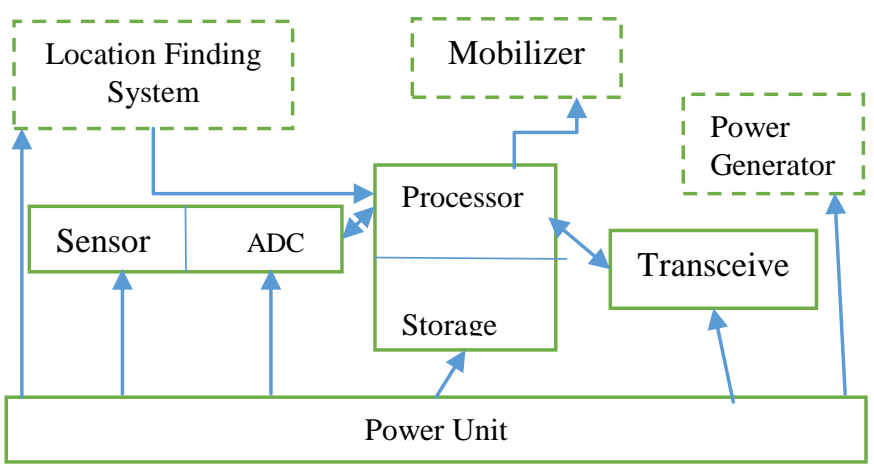

Figure 2 Major parts of a sensor node

They might likewise have application subordinate extra parts, for example, an area discovering system, a power generator and a mobilizer. The sensors deliver the analog signals and ADC convert them to digital signals, and served to the processing unit. A small storage unit is associated with processing unit and achieves the procedures that make the sensor node to cooperate with the other nodes to carry out the given sensing tasks. The transceiver unit associate the nodes to the network. The most vital part is the power unit. Power units may be upheld by a force looking unit, for example, sun based cells. The sensed data must be conveyed to a control center called Base Station (BS). Just the highly energized nodes can impart data to the BS. The sink (Base Station) communicates with the client through web or satellite correspondence. It is spotted close to the sensor field or decently prepared hubs of the sensor system [2]. The sensor applications can be sorted into information assembling or 
tracking applications. Information gathering applications use sensor nodes to intermittently measure the estimation of a specific ecological variable and recorded qualities are gathered by a sink for further preparing and tracking applications consistently screen the earth for the vicinity of signs which can particularly recognize an item being followed [15]. Sensor systems can be utilized as a part of military applications (focusing on, fight harm evaluation), therapeutic applications (drug organization in doctor's facilities by appending sensors with meds, following specialists and patients in clinics), natural applications (surge identification, woods fire location, contamination study) and home applications (catching auto robberies, vehicle following and discovering). It is expected that all nodes of a system live on a plane in 2D WSN outline of physical systems. This supposition is not legitimate if a system is conveyed in space or sea, where nodes of a system are disseminated over a 3D space. For instance, 2D configuration of submerged sensor system is not fitting; it obliges 3D outline [4].

\subsection{Underwater Wireless Sensor Network}

UWSNs are altogether dissimilar from existing systems because of the inherent properties of the submerged situations [5]. The submerged sensor system comprises of sensor gadgets, base stations, surface station and onshore sink. Sensor gadgets will sense the items inside the water and will pass the signals to the base station. The sensor nodes deployed at the ocean bottom can't communicate with nodes closer to the surface; they need multi-hop communication upheld by proper routing plan. At last, the estimations are collected at a satellite from all surface sinks [26].

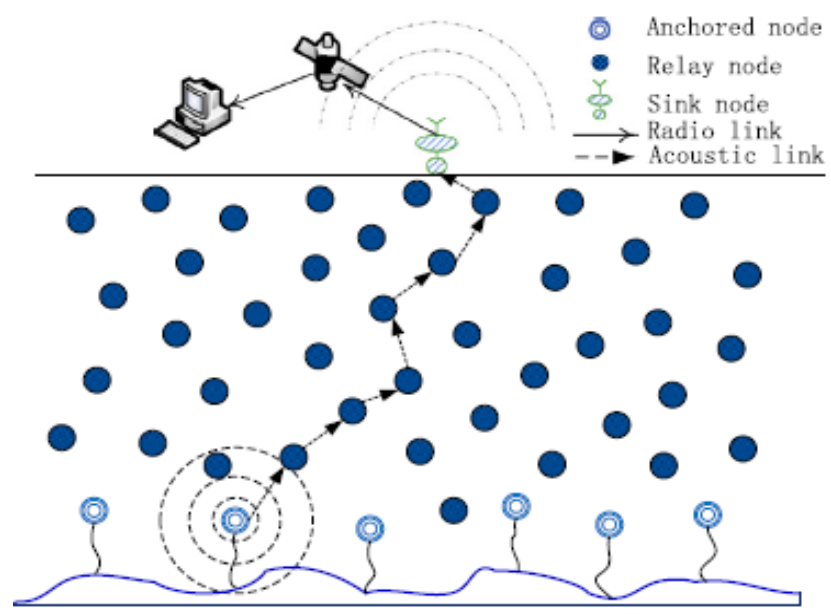

Figure 3 Architecture of the mobile UWSN [25]

The authors in [16] suggested two correspondence architectures, i.e., two-dimensional and three-dimensional. In two-dimensional building design, sensor nodes are settled at the bottom where these can be sorted out in clusters and are interrelated with one or more surface gateways by method for acoustic connections. The surface gateways are in charge of transmitting information from sea base to surface sink. In three-dimensional structural planning, sensor nodes are deployed at various depth levels covering the whole volume being observed. Since the organization of the sensor nodes is extremely troublesome and some of the time it is carried out physically by setting the sensor nodes at predetermined areas [29]. Nodes are appended with surface floats through wires and their lengths can be managed to modify the height of the sensor nodes. The high error rates of submerged connections of nodes could have drained their energy resources [27].
Radio signal is generally utilized as remote transmission media as a part of the physical sensor system, yet in submerged remote sensor systems, radio signs can't function admirably because of fast weakening, bringing about short propagation delay [25]. The submerged sensor systems send the utilization of acoustic signs in light of the fact that these signs have high propagation delays and require low transmission capacity. Because of different components of submerged situations, node versatility turns into a nonunimportant issue [28].

\section{RELATED WORK}

The ground-based wireless sensor networks and underwater wireless sensor networks are distinguished from each other. In [1], Akyildiz, Ian F. et al (2002) has described the concept of the sensor networks and has explored the applications of the sensor networks and a list of factors are provided that affect the design of the wireless sensor networks. In [2], Al-Obaisat Yazeed and Robin Braun (2007) have presented the architecture and design features of the wireless sensor networks and also discussed the design goals and challenges for routing protocols. In [3], Pal S. et al. (2010) categorized the routing protocols on the basis of a number of factors and summarized them on the basis of their operation mode and a comparative analysis of the routing protocols is also presented. In [4], Roy S. et al. (2012) has proposed a framework for topology construction of 3D WSN using computational geometry for a given $3 \mathrm{D}$ space monitoring application. In [5], Domingo, Mari Carmen, and Rui Prior measured the total energy consumption in underwater sensor networks and two different situations are considered and various functioning principles for routing protocols are proposed. In [6], Ayaz M. et al. (2012) has proposed a dynamic address based routing protocol for handling the problem of node mobility. In [7], Bayrakdar Y. et al. (2011) identified the existing routing protocols shortcomings. In [8], Yan H. et al. (2008) has proposed a protocol called DBR that encompasses the use of depth of the sensor nodes. In [9], Liang W. et al. (2007) has proposed ICRP routing protocol and evaluate its performance. In [10], Mohsin Raza Jafri et al. (2014) has proposed Delay-Sensitive Depth-Based Routing (DSDBR), Delay-Sensitive Energy Efficient Depth-Based Routing (DSEEDBR) and Delay-Sensitive Adaptive Mobility of acoustic nodes in Threshold-optimized Depth-based routing (DSAMCTD) protocols to allow the depth-based routing schemes. In [11], Mahmood S. et al. (2014) has extended the DBR protocol by limiting the number of forwarding nodes and have extended the network lifetime and energy consumption of the DBR. In [12], Ayaz M. et al (2009) has proposed a dynamic address based routing protocol. In [13], Mahapatro G. et al. (2012) has proposed depth based multi hop routing. In [14], Singh M. P. et al. (2010) presented a comparative study of the strengths and the weaknesses of the routing algorithms. In [15], Siddharth Ramesh (2008) has given the protocol architecture of the wireless sensor networks. In [16], Akyildiz et al. have discussed different architectures of underwater wireless sensor networks architecture. In [17], Liang et al. proposed information carrying routing protocol.In [18], Akkaya, Kemal, and Andrew Newell (2009) proposed a node deployment method which helps to increase the initial network coverage in an iterative basis.In [19], Ayaz, Muhammad, and Azween Abdullah proposed a hop by hop dynamic address based routing protocol and achieved the higher data deliveries with optimum delays and energy consumptions. In [20], authors have proposed a directional flooding based routing protocol called DFR. In [21], Guangzhong, Liu, and $\mathrm{Li}$ Zhibin 
provided a new routing protocol DBMR based on DBR. In [22], Shao et al (2014) presented a new routing protocol DBURP for underwater wireless sensor networks. In [23], Jiang et al. (2006) explored major four categories of routing protocols. In [24], Ramesh, Siddharth presented a protocol architecture of wireless sensor networks. In [25], Yu et al (2014)proposed an adaptive hop-by-hop vector-based forwarding routing protocol on the basis of HH-VBF (called AHH-VBF). In [26], Yick et al (2008) built a more authentic signal irregularity model, which can be collapsed into a variety of special cases easily, and three representative topology control objectives. In [27], Mota et al (2014) presented an overview on opportunistic networks. In [28], Liu et al (2012) constructed a mobility model for UWSNs nodes and attained various topology control objectives and also designed distributed radius determination algorithm.

\section{ROUTING PROTOCOLS FOR UWSNs}

Directing procedures are needed for sending information among sensor nodes and the base stations for correspondence. Directing conventions are classified on the premise of:

1. Network Architecture

2. Data Sending

3. Protocol Operation

The authors further categorize the protocols in following categories: On the basis of network architecture, routing protocols are categorized as location based routing, flat routing, hierarchical routing. On the basis of data forwarding, routing protocols are categorized in multi-path routing, single path routing. On the basis of protocol operation, routing protocols are classified in source initiated routing, table driven routing and data aggregation. Hierarchical routing is utilized to achieve energy effective routing by utilizing higher energized nodes for processing and sending the data whereas low energized nodes are utilized to perform sensing the zone of interest. The fundamental point of various hierarchical directing procedure is to effectively keep up the energy utilization of sensor nodes by including them in multi-hop correspondence inside a cluster and data aggregation and fusion are performed by keeping in mind the end goal to diminishing the quantity of transferred messages to the sink [23]. The flat protocols make use of the technique that any node that has data to be sent, first searches a legal path to the BS and then sends data. Nodes near the base station rapidly drain out their energy. Location based routing protocols need location data of the sensor nodes. Location based routing protocols can be utilized as a part of systems where sensors have the capacity to focus their positions utilizing a variety of localization algorithms [22]. The directing procedure arranges the location information of sensor nodes to ascertain the separation among two specific nodes to estimate energy utilization [14].

\subsection{Information Carrying Routing \\ Protocol (ICRP)}

A large number of the routing protocols makes use of separate packets for control data and information communication. In [17], authors proposed Information Carrying Routing Protocol (ICRP) with a specific end goal to identify the routing issue for submerged communications. For energy efficient and scalable routing, ICRP makes use of control packets that are carried by data packets. ICRP does not incorporate the use of state or location information, and also only a small fraction of the nodes participate in the routing process. The ICRP incorporates three steps that are, route finding, route preservation and route renunciation [9]. The route finding step is launched by the source of data packets. When the source node will direct the packet to the destination node however there is no occurrence of route, it will broadcast the packets conveyingroute finding data and alternate node will further pass the packets and notethereverse path. The destination node gets the opposite path from source node to destination as the destination node gets packet. In the event that destination node has information packet to source node, the acknowledgement message can be conveyed by information packet and information packet can be conveyed along the reverse path. Otherwise, an acknowledgement packet is transmitted to source node through the opposite path by destination node. With route discovery step, one path is associated with every destination node, every path have a period property, the interval property indicates the time that the opposite route is not utilized for transmission and known as route lifetime. The bigger value of route lifetime indicates that the path is not taken in use for a long time. At the point when the lifetime surpasses the threshold value, the route becomes illegal and node needs route rediscovery. As soon as node uses the way below its threshold value, the lifetime is reset to 0 , indicating route is utilized quite recently. In the third step, when the path lifetime parameter in routing table surpasses the characterized threshold value, the path is viewed as illegal and is dropped. When there are packets to be sent to the destination, the routerediscovery is required and route table things areredesigned. Basic routing mechanism has some execution issues. To start with, when a node does not find any route to send data to a specified destination, it will broadcast the information packet. The wastage of node energy is due to more occurrence of broadcasts that diminishes the life of the entire system. Also, every path is associated with expiry time that can be extremely sensitive for delivery ratios.

\subsection{Depth Based Routing Protocol}

Since location based routing schemes make use of location data of the sensor nodes in the system, which is a challenging assignment for UWSNs, DBR needs just the depth information of sensor nodes. DBR is a desirous algorithm that tries to direct a packet from a source node to sinks [13]. To acquire the depth of current node, each sensor node is outfitted with a reasonable depth sensor. DBR utilizes the various sink construction modeling as a part of which different number of sinks are put on the water level and are utilized to gather the information packets delivered by the sensor nodes. DBR takes the routing decision on the basis of depth data, and advances the information packets from higher depth nodes to lower depth sensor nodes. When a node has aninformation packet that is to be sent, it will first sense its present depthposition in respect to the surface and encapsulate it in the packet header and after that broadcast it. The receiving node will forward this packet by first calculating its depth position and if its depth is smaller than the value encapsulated in the packet, it will basically avoid the packet. Packets got at any of the information sink are considered as effective conveyance at the last destination and these information sinks can correspond productively through radio channel. The primary points of interest of DBR are as per the following. 1) It doesn't need location data. 2) It deals with dynamic network systems with great energy productivity. 3) It exploits numerous sink system construction modeling without presenting additional expense [8]. Be that as it may, it has a few genuine issues. Initially, DBR has just greedy mode, which is not ready to attain to high conveyance proportions in sparse territories. Second, sending the information packets in broadcast way can diminish the execution of the system. Third, it utilizes the flooding mode to send information that 
causes a substantialnumber of repetitive information sending and channel inhabitance [21].

\subsection{Hop- by- Hop Dynamic Address based Routing Protocol}

The greater part of the routing protocols assumed that location data of entire system is accessible and each node ought to be furnished with depth or weight sensor, which expand the expense of the system as well as turn into a load on the discriminating node energy. The authors in [19] have proposed a dynamic addressing based routing protocol named $\mathrm{H} 2 \mathrm{H}-\mathrm{DAB}$ and it does not take into consideration as the greater part of the protocols. Sensor nodes utilize the dynamic address to get new delivers as indicated by their new positions at distinctive depth levels. This convention utilizes numerous surface buoys that are utilized to gather information and a few nodes are secured at bottom and the rest of the nodes are tied down at diverse depths [6]. Nodes closer to the surface have smaller value of addresses, and these addresses get to be bigger as the nodes travel towards the bottom. H2H-DAB finishes its undertaking in two stages. In first stage, it assigns the dynamic addresses to the sensor nodes, and in second stage, information is sent utilizing these addresses. With the assistance of hello packets, dynamic addresses are assigned to nodes and these addresses are produced by the surface sinks. Upper layer nodes get the packets form the nodes that receives the packets first in an avaricious way. Packets arriving at any of the sinks will be considered as conveyed effectively. $\mathrm{H} 2 \mathrm{H}-$ DAB has numerous favorable circumstances [12]: it doesn't oblige any particular equipment, no dimensional locational data obliged and node developments can be taken care of effectively without keeping up mind complex routing tables. The impediment of this convention is: while picking a forwarder node, the source does not get a reaction from its neighbors particularly in sparse systems. This issue can be resolved by sitting tight for a certain time and afterward advances the packet to a neighbor having same depth [7].

\subsection{Directional Flooding based Routing Protocol}

There is much overhead involved in path establishment as control messages and in addition existing routing protocols did not consider the connection quality. To expand the dependability, in [20], the authors proposed the DirectionalFlooding-Based routing (DFR) protocol. This protocol takes into consideration the location information of nodes and its nearest neighbors as well as last destination and a small quantity of sensor nodes participate in the same for a particular packet so as to counteract multicasting over the entire system, and sending nodes are chosen by connection quality.

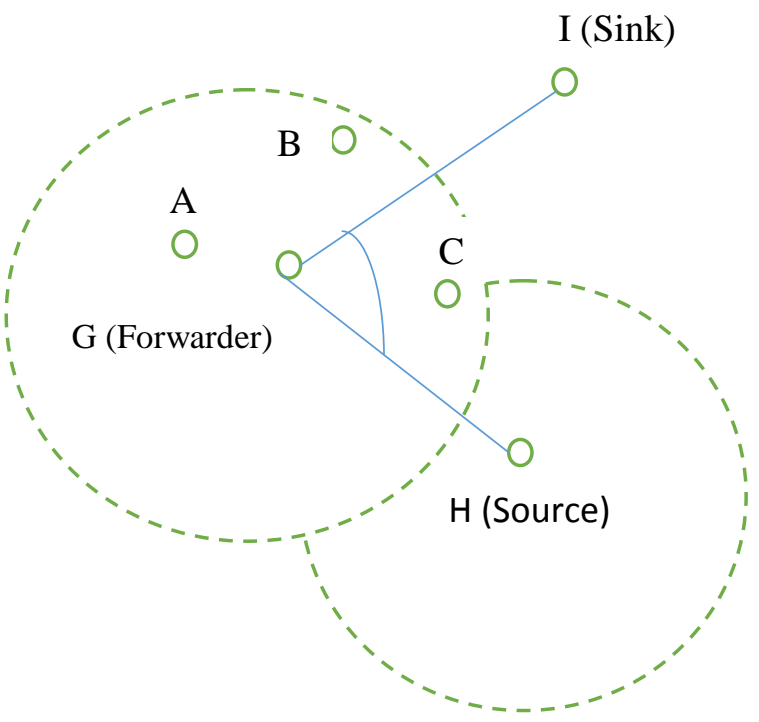

Figure 4 Example of transmission in DFR

As indicated in Fig. 4, the broadcasting zone is chosen by the plot in the middle of GS and GD, where $G$ is the packet getting node, while $\mathrm{H}$ and $\mathrm{I}$ represent the source and destination nodes, separately. In the wake of accepting an information packet, $\mathrm{G}$ decides powerfully the packet sending by contrasting HGD and a standard plot for flooding, called BASE_ANGLE, which is incorporated in the got packet. In order to handle the high and dynamic packet error rate, BASE_ANGLE is adjusted in a hop-by-hop manner and dynamically find a flooding zone. DFR execution relies on upon the quantity of nodes after the process of flooding the information packet and are chosen as the next hop.

\subsection{Constraint based Depth based routing protocol}

In DBR protocol, there are removed transmissions between the sensor nodes particularly in the medium-depth regions that leads to extensive propagation delay [10]. The significant deficiencies of the DBR are:

- $\quad$ Large propagation delay.

- $\quad$ More energy utilization.

- Unnecessary information sending.

These deficiencies are evacuated by the authors of [11]. They proposed an expansion form of DBR called constraint based depth based routing protocol (CDBR). The sensor nodes are sent under the water haphazardly. Various sinks are sent on the sea level whereas the sensor nodes are in charge of conveying the sensed information to the sinks. RF modems and Acoustic modems are the major parts of the sinks. The sensor nodes under the water are furnished with Acoustic modems. The nodes correspond with one another and the Sinks utilizing the Acoustic Modems. The sinks correspond with one another and the on-shore server farm utilizing the RF Modems. Information arriving at any of the sinks is considered as information conveyed effectively. It is additionally expected that the sensor nodes are outfitted with depth sensors which can be utilized to know the depth data. The protocol comprises of two stages:

- Optimal forwarder node set selection

- $\quad$ Forwarding node selection

Optimal forwarder node set selection:

This stage begins with the ID of the neighbors of the source node. The nodes having depth lower than the depth of source node are distinguished as its neighbors. The quantity of 
neighboring nodes is further obliged by applying a worldwide parameter called depth threshold. This permits just those nodes to get the information which are at a depth difference more than depth threshold. Depth threshold is characterized as subtraction of depth of source node and the depth of neighboring node. Among the recognized neighbors, source node distinguishes a set of nodes known as an ideal forwarder node set. In the neighbor recognizable proof stage, it is vital to know whether the Source is inside the scope of any sink or not. In the event that a sink is in its nearby area, the information is conveyed straightforwardly to the sink. In the event that there is no sink in the scope of source node, then it is sent to its next hop forwarder node set. At long last one node out of this node set is chosen to telecast information to the next hop forwarder node set.

\section{Forwarding node selection:}

In this stage, the source node first distinguishes a set of nodes in its transmission range known as ideal forwarder node set. All the nodes in this set get the sensed data telecasted by source node. In CDBR among the forwarder node set, a node with least depth is chosen for information forwarding.

\subsection{Vector based Forwarding}

Vector based routing protocol handles the problem of maintenance and recovery of the routes due to constant node movements. VBF does not take into consideration the information regarding to state of the nodes and only a small quantity of nodes are involved in the forwarding process. Packet loss and node failure problems are reduced by sending the packets along most used routes. This protocol takes into account the information about location of source and destination and is encapsulated in packet. The concept of a vector is same as virtual routing pipe through which packets are sent. Packets are forwarded with the help of nodes that are at short distances form the vector and this leads to reduced network traffic and the dynamic topology can be managed easily. VBF has some confinements. To begin with, the routing efficiency of the network is affected by the formation of vector. Second, the routing performance is affected by vector radius threshold. Third, ample communication overhead is present due to its 3-way handshake nature.

\subsection{Sector-based Routing with Destination Location Prediction (SBR-DLP)}

The SBR-DLP takes into account the location information and route a data packet in completely versatile underwater wireless sensor networks. Sensor nodes need not to carry the information about neighbor. Data packets are sent in a hopby- hop manner. As indicated in Fig. 5, a node F wants to send data packet to destination $\mathrm{S}$. This can be accomplished by discovering its next hop by broadcasting a Chk_Ngb packet, and this packet encapsulates its present position and packet ID. The node that gets Chk_Ngb will investigate whether its position is near to the destination node $\mathrm{S}$ than the length between nodes $F$ and $S$. The node $F$ will get a reply form a node that satisfy the above condition and this can be accomplished by sending a Chk_Ngb_Reply packet.

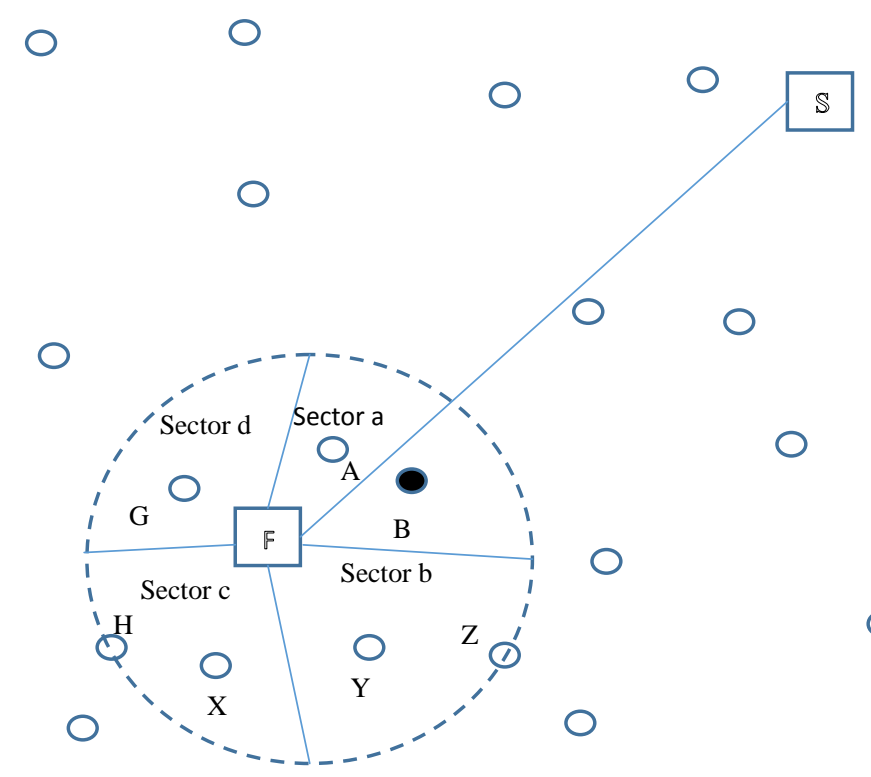

Figure 5 The process of forwarding node selection at the sender

Destination portability is reduced by supposing the preplanned movements that are wholly known to all the sensor nodes before their deployment. This assumption arises two issues. First, the flexibility of the network is reduced. Second, the destination node is moved away from its planned movements.

\subsection{Mobile delay-tolerant approach (DDD)}

The more energy consumption for acoustic channels need energy saving that becomes more critical in underwater wireless sensor networks. Authors proposed a Delay-tolerant Data Dolphin (DDD) scheme for delay-tolerant applications to upturn the energy efficiency. DDD uses collector nodes called dolphins to gather information that is sensed by the sensor nodes. The recommended scheme shuns multi-hop communication, and data is sent to acoustic nodes that are in its communication range. The sensors occasionally wakeup to sense data and to generate some events. The acoustic modem is centered on two parts. The first part is utilized for acoustic communication with the close dolphin, and the other part is a low-power transceiver used to determine the occurrence of dolphin nodes and the activation of the first component is done through this. The dolphins can float either with arbitrary or measured mobility as per network condition. A dolphin announces its presence by broadcasting the signal. Advertising period $t$ can be balanced by range $r$ of sensor nodes, and the speed of dolphin v. As soon as dolphins reach a base station on the surface, they deliver packets to it. The performance of DDD is regulated by the number of dolphin nodes. If the dolphin nodes are not as much required, the gathering process will not be accomplished. If 7 dolphins are used for 25 sensor nodes, then cost will become a chief problem.

\subsection{Location- aware Source Routing}

LASR makes use of two techniques to handle latency of acoustic channel: link quality metric and location awareness. LASR uses expected transmission count (ETX) where moreinformed decisions are provided by link quality. The incoming transmissions helps to get location information and provide a guideline to estimate local network topology. The current location of other nodes can be guessed with the 
assistance of tracking system while all the information of the network along with the routes and topology information are encapsulated in the protocol header. LASR relies on source routing technique. The packet header continues to increase with the increase in hop count and this leads to acoustic communication overhead. The usage of ETX as link quality is not easy for UWSN because it assumes symmetrical links and link quality to be same in both directions.

\section{COMPARATIVE ANALYSIS OF ROUTING PROTOCOLS FOR UWSNS}

\begin{tabular}{|c|c|c|c|c|c|c|c|}
\hline $\begin{array}{l}\text { Parameter } \\
\text { And Protocol }\end{array}$ & $\begin{array}{l}\text { Localization of } \\
\text { nodes required }\end{array}$ & $\begin{array}{l}\text { Multi- sink } \\
\text { architecture }\end{array}$ & Technique used & $\begin{array}{l}\text { Basic } \\
\text { parameter } \\
\text { onto which } \\
\text { routing } \\
\text { decision is } \\
\text { made }\end{array}$ & $\begin{array}{l}\text { Network } \\
\text { topology }\end{array}$ & $\begin{array}{l}\text { Control } \\
\text { packets }\end{array}$ & $\begin{array}{l}\text { Routing } \\
\text { table } \\
\text { required }\end{array}$ \\
\hline ICRP & No & No & Broadcasting & Path life- time & Dynamic & Yes & Yes \\
\hline DFR & Yes & No & Packet flooding & $\begin{array}{l}\text { Base angle or } \\
\text { criterion angle }\end{array}$ & Dynamic & No & Yes \\
\hline DBR & $\begin{array}{l}\text { Only depth } \\
\text { information }\end{array}$ & Yes & Broadcasting & $\begin{array}{l}\text { Depth of } \\
\text { neighbor }\end{array}$ & Static & No & Yes \\
\hline CDBR & $\begin{array}{l}\text { Only depth } \\
\text { information }\end{array}$ & Yes & Broadcasting & $\begin{array}{l}\text { Depth } \\
\text { threshold } \\
\text { value }\end{array}$ & Static & No & Yes \\
\hline H2- DAB & No & Yes & $\begin{array}{l}\text { Dynamic } \\
\text { addressing }\end{array}$ & $\begin{array}{l}\text { Dynamic } \\
\text { address }\end{array}$ & Dynamic & Yes & No \\
\hline VBF & Yes & No & $\begin{array}{l}\text { Virtual routing } \\
\text { pipe }\end{array}$ & $\begin{array}{l}\text { Node nearer } \\
\text { to the virtual } \\
\text { pipe }\end{array}$ & Dynamic & No & Yes \\
\hline SBR-DLP & Yes & No & Multicasting & $\begin{array}{l}\text { Distance to } \\
\text { destination } \\
\text { node }\end{array}$ & Static & No & Yes \\
\hline DDD & No & Yes & Broadcasting & N/A & Static & Yes & No \\
\hline LASR & Yes & No & $\begin{array}{l}\text { Link quality } \\
\text { metric and } \\
\text { location } \\
\text { awareness }\end{array}$ & $\begin{array}{l}\text { Shortest path } \\
\text { metric }\end{array}$ & Local & No & Yes \\
\hline
\end{tabular}

\section{CONCLUSION}

In this paper, many routing protocols has been considered that are used in underwater wireless sensor networks and some of these routing protocols need location information while some need only depth information and some do not need any location information. A comparative analysis is also done on the basis of various parameters like use of control packets, network topology, localization information etc. There are many research challenges that are needed to be resolved like how many surface sinks should be deployed for predetermined quantity of sensor nodes and to extend the static network topology to dynamic network topology. This work has not considered any improvement over the existing DBR protocol, so in near future we will modify the DBR routing protocol using the particle swarm optimization approaches. The use of particle swarm optimization guarantee the shortest path in less amount of time therefore we will include the overall performance of DBR protocol.

\section{REFERENCES}

[1] Akyildiz, Ian F., Weilian Su, Yogesh Sankarasubramaniam, and Erdal Cayirci. "Wireless sensor networks: a survey." Computer networks 38, no. 4 (2002): 393-422.

[2] Al-Obaisat, Yazeed, and Robin Braun. "On wireless sensor networks: architectures, protocols, applications, and management." (2007).

[3] Pal, Subhajit, Debnath Bhattacharyya, Geetam S. Tomar, and Tai-hoon Kim. "Wireless sensor networks and its 
routing protocols: a comparative study." InComputational Intelligence and Communication Networks (CICN), 2010 International Conference on, pp. 314-319. IEEE, 2010.

[4] Roy, Sarbani, and Nandini Mukherjee. "Topology Construction of 3D Wireless Sensor Network." In Advances in Computing and Information Technology, pp. 533-542. Springer Berlin Heidelberg, 2012.

[5] Domingo, Mari Carmen, and Rui Prior. "Energy analysis of routing protocols for underwater wireless sensor networks." Computer Communications 31, no. 6 (2008): 1227-1238.

[6] Ayaz, Muhammad, Azween Abdullah, Ibrahima Faye, and Yasir Batira. "An efficient dynamic addressing based routing protocol for underwater wireless sensor networks." Computer Communications 35, no. 4 (2012): 475-486.

[7] Bayrakdar, Yonca, Nirvana Meratnia, and Aylin Kantarci. "A comparative view of routing protocols for underwater wireless in sensor networks." In OCEANS, 2011 IEEE-Spain, pp. 1-5. IEEE, 2011.

[8] Yan, Hai, Zhijie Jerry Shi, and Jun-Hong Cui. "DBR: depth-based routing for underwater sensor networks." NETWORKING 2008 Ad Hoc and Sensor Networks, Wireless Networks, Next Generation Internet, pp. 72-86. Springer Berlin Heidelberg, 2008.

[9] Liang, Wei, Haibin Yu, Lin Liu, Bangxiang Li, and Chang Che. "Information-carrying based routing protocol for underwater acoustic sensor network." InMechatronics and Automation, 2007. ICMA 2007. International Conference on, pp. 729-734. IEEE, 2007.

[10] Jafri, Mohsin Raza, Muhammad Moid Sandhu, Kamran Latif, Zahoor Ali Khan, Ansar Ul Haque Yasar, and Nadeem Javaid. "Towards delay-sensitive routing in underwater wireless sensor networks." Procedia Computer Science 37 (2014): 228-235.

[11] Mahmood, S., H. Nasir, S. Tariq, H. Ashraf, M. Pervaiz, Z. A. Khan, and N. Javaid. "Forwarding Nodes Constraint based DBR (CDBR) and EEDBR (CEEDBR) in Underwater WSNs." Procedia Computer Science 34 (2014): 228-235.

[12] Ayaz, Muhammad, and Azween Abdullah. "Hop-by-hop dynamic addressing based (H2-DAB) routing protocol for underwater wireless sensor networks." InInformation and Multimedia Technology, 2009. ICIMT'09. International Conference on, pp. 436-441. IEEE, 2009.

[13] Mahapatro, Gurucharan, and U. Devee Prasan. "Energy Efficient Multiple Sink Variation To The Depth-Based Route Protocol For Under Water Sensor Network." (2012).

[14] Singh, Shio Kumar, M. P. Singh, and D. K. Singh. "Routing protocols in wireless sensor networks-A survey." International Journal of Computer Science \& Engineering Survey (IJCSES) Vol 1 (2010): 63-83.

[15] Ramesh, Siddharth. "A Protocol Architecture for Wireless Sensor Networks." In Proc. of the 1st ACM International Workshop on Wireless Sensor Networks and Applications (WSNA). 2008.
[16] Akyildiz, Ian F., Dario Pompili, and Tommaso Melodia. "Underwater acoustic sensor networks: research challenges." Ad hoc networks 3, no. 3 (2005): 257-279.

[17] Liang, Wei, Haibin Yu, Lin Liu, Bangxiang Li, and Chang Che. "Information-carrying based routing protocol for underwater acoustic sensor network." In Mechatronics and Automation, 2007. ICMA 2007. International Conference on, pp. 729-734. IEEE, 2007.

[18] Akkaya, Kemal, and Andrew Newell. "Self-deployment of sensors for maximized coverage in underwater acoustic sensor networks." Computer Communications 32, no. 7 (2009): 1233-1244.

[19] Ayaz, Muhammad, and Azween Abdullah. "Hop-by-hop dynamic addressing based (H2-DAB) routing protocol for underwater wireless sensor networks." In Information and Multimedia Technology, 2009. ICIMT'09. International Conference on, pp. 436-441. IEEE, 2009.

[20] Hwang, Daeyoup, and Dongkyun Kim. "DFR: Directional flooding-based routing protocol for underwater sensor networks." In OCEANS 2008, pp. 1-7. IEEE, 2008.

[21] Guangzhong, Liu, and Li Zhibin. "Depth-based multihop routing protocol for underwater sensor network." In Industrial Mechatronics and Automation (ICIMA), 2010 2nd International Conference on, vol. 2, pp. 268-270. IEEE, 2010.

[22] Shao, Deo Donald, Mohammed Yaqub, Haitham AM Raid, A. EL-Ghareeb, Hossein Parvan, Ebrahim Behrouzian Nejad, Seyed Enayatolah Alavi et al. "Leveraging on Mobile Telephony to Strengthen Child Birth Registration Process in Tanzania: Proposed Prototype." International Journal Of Computer Science And Network Solutions (2014).

[23] Jiang, Peng, Yu Wen, Jianzhong Wang, Xingfa Shen, and Anke Xue. "A study of routing protocols in wireless sensor networks." In Intelligent Control and Automation 2006. WCICA 2006. The Sixth World Congress on, vol. 1, pp. 266-270. IEEE, 2006.

[24] Ramesh, Siddharth. "A Protocol Architecture for Wireless Sensor Networks." In Proc. of the 1st ACM International Workshop on Wireless Sensor Networks and Applications (WSNA). 2008.

[25] Yu, Haitao, Nianmin Yao, and Jun Liu. "An adaptive routing protocol in underwater sparse acoustic sensor networks." Ad Hoc Networks (2014).

[26] Yick, Jennifer, Biswanath Mukherjee, and Dipak Ghosal. "Wireless sensor network survey." Computer networks 52, no. 12 (2008): 2292-2330.

[27] Mota, Vinícius FS, Felipe D. Cunha, Daniel F. Macedo, José MS Nogueira, and Antonio AF Loureiro. "Protocols, mobility models and tools in opportunistic networks: A survey." Computer Communications 48 (2014): 5-19.

[28] Liu, Linfeng, Ruchuan Wang, and Fu Xiao. "Topology control algorithm for underwater wireless senso networks using GPS-free mobile sensor nodes." Journal of Network and Computer Applications 35, no. 6 (2012): 1953-1963. 